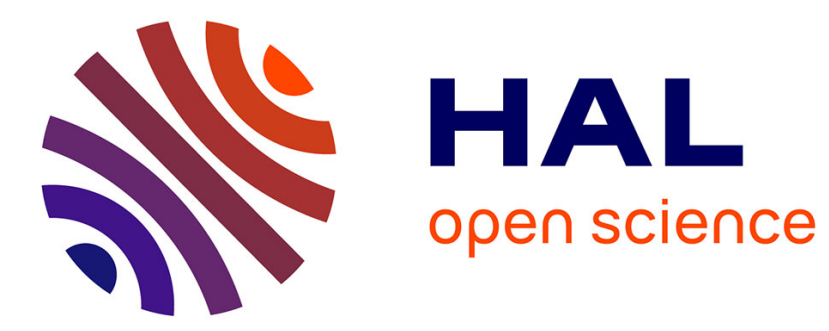

\title{
Contribution à la dosimétrie des fluences de neutrons thermiques
}

\author{
M. Maurette, J. Mory
}

\section{To cite this version:}

M. Maurette, J. Mory. Contribution à la dosimétrie des fluences de neutrons thermiques. Revue de Physique Appliquée, 1968, 3 (3), pp.209-215. 10.1051/rphysap:0196800303020900 jpa-00242849

\section{HAL Id: jpa-00242849 https://hal.science/jpa-00242849}

Submitted on 1 Jan 1968

HAL is a multi-disciplinary open access archive for the deposit and dissemination of scientific research documents, whether they are published or not. The documents may come from teaching and research institutions in France or abroad, or from public or private research centers.
L'archive ouverte pluridisciplinaire HAL, est destinée au dépôt et à la diffusion de documents scientifiques de niveau recherche, publiés ou non, émanant des établissements d'enseignement et de recherche français ou étrangers, des laboratoires publics ou privés. 


\title{
REVUE DE PHYSIQUE APPLIQUÉE
}

\author{
Supplément au "Journal de Physique "
}

\section{GONTRIBUTION A LA DOSIMÉTRIE DES FLUENGES DE NEUTRONS THERMIQUES}

\author{
Par M. MAURETTE (1) et J. MORY ( $\left.{ }^{2}\right)$. \\ (Reçu le 5 avvil 1968.)
}

\begin{abstract}
Résumé. - On a utilisé l'enregistrement des trajectoires de fragments de fission dans le verre et le mica, et celui des particules $\alpha$ dans le nitrate de cellulose, pour mesurer des fluences de neutrons thermiques. On a ainsi obtenu une série de dosimètres dont le prix de revient est négligeable et qui sont calibrés à $\pm 10 \%$ pour les mesures absolues de fluences comprises entre $10^{6}$ et $10^{21} \mathrm{n} / \mathrm{cm}^{2}$; dans ce domaine, les dosimètres permettent d'effectuer des mesures relatives de fluences très précises.
\end{abstract}

\begin{abstract}
The recording of fission fragment trajectories in glass and mica and of $\alpha$ particles in cellulose nitrate has been used for the measurement of thermal neutron fluxes. A series of very cheap dosimeters, calibrated at $\pm 10 \%$ for the measurement of absolute flux values between $10^{6}$ and $10^{21} \mathrm{n} / \mathrm{cm}^{2}$ have been realized; in this range of fluxes, the dosimeters can provide relative flux values with great accuracy.
\end{abstract}

Introduction. - On sait, depuis les travaux de Silk et Barnes [1] et de Price et Walker [2], qu'un grand nombre de solides isolants peuvent enregistrer, sous forme de traces révélables par attaque chimique, les trajectoires d'ions suffisamment lourds et énergiques.

Cette caractéristique a déjà été utilisée pour mesurer certaines fluences de neutrons thermiques [3-10]. Les dosimètres comprennent un convertisseur dans lequel les neutrons sont transformés en particules ionisantes décelables au moyen de réactions nucléaires auxiliaires, et un détecteur qui enregistre les ions issus du convertisseur. L'élément convertisseur est présent soit dans la masse du détecteur lui-même, soit sous forme d'une couche mince évaporée ou d'une feuille épaisse appliquée à la surface du détecteur. Nous verrons que la densité $\rho$ (en nombre $/ \mathrm{cm}^{2}$ ) des traces enregistrées dans le détecteur est proportionnelle à la valeur $\varphi\left(\mathrm{en} \mathrm{n} / \mathrm{cm}^{2}\right)$ de la fluence de neutrons; il est donc possible de connaître la valeur de $\varphi=(1 / \tau) \cdot \rho$, en mesurant $\rho$ avec un microscope optique, à condition d'avoir déterminé $\tau$ au cours d'une expérience de calibration $(\tau$ est le nombre de traces produites par neutron incident, ou encore le taux de production de traces).

(1) Laboratoire de Chimie-Physique de la Faculté des Sciences de Paris, Centre d'Orsay.

(2) S.E.C.P.E.R., Centre d'Etudes Nucléaires de Fontenay-aux-Roses.
Les différents travaux publiés dans ce domaine ont jusqu'ici porté :

- Sur la mesure de fluences de neutrons thermiques dans des domaines restreints au moyen des traces induites par les éléments fissiles, soit contenus dans les isolants eux-mêmes $[3,7]$, soit appliqués en sources extérieures contre ces isolants $[4,5,6,8,10]$;

- Sur la mesure, surtout dans des buts de radioprotection, de certaines gammes de fluences de neutrons rapides $[3,6,8]$;

- Sur la mesure de la répartition spatiale des neutrons thermiques [7,9].

Le but esseņtiel de notre travail a été de mettre au point une série de dosimètres de neutrons thermiques « à traces " très simples, robustes, peu coûteux, susceptibles d'être préparés et utilisés sans connaissances particulières et avec un appareillage simple, pour mesurer des fluences de neutrons thermiques comprises entre $10^{6}$ et $10^{21} \mathrm{n} / \mathrm{cm}^{2}$; nous désirions également faire des mesures relatives de fluences aussi précises que possible.

I. Description des dosimètres. - Le constituant de base de nos dosimètres est le verre, qui contient soit de l'uranium, soit du bore. Sous l'effet des neutrons thermiques, l'uranium subit la fission. Quant au bore 10, contenu dans le bore naturel, il émet, par la réaction ${ }^{10} \mathrm{~B}(\mathrm{n}, \alpha)^{7} \mathrm{~J} \mathrm{i}$, une particule $\alpha$ de $1,7 \mathrm{MeV}$ 


\section{TABLEAU I}

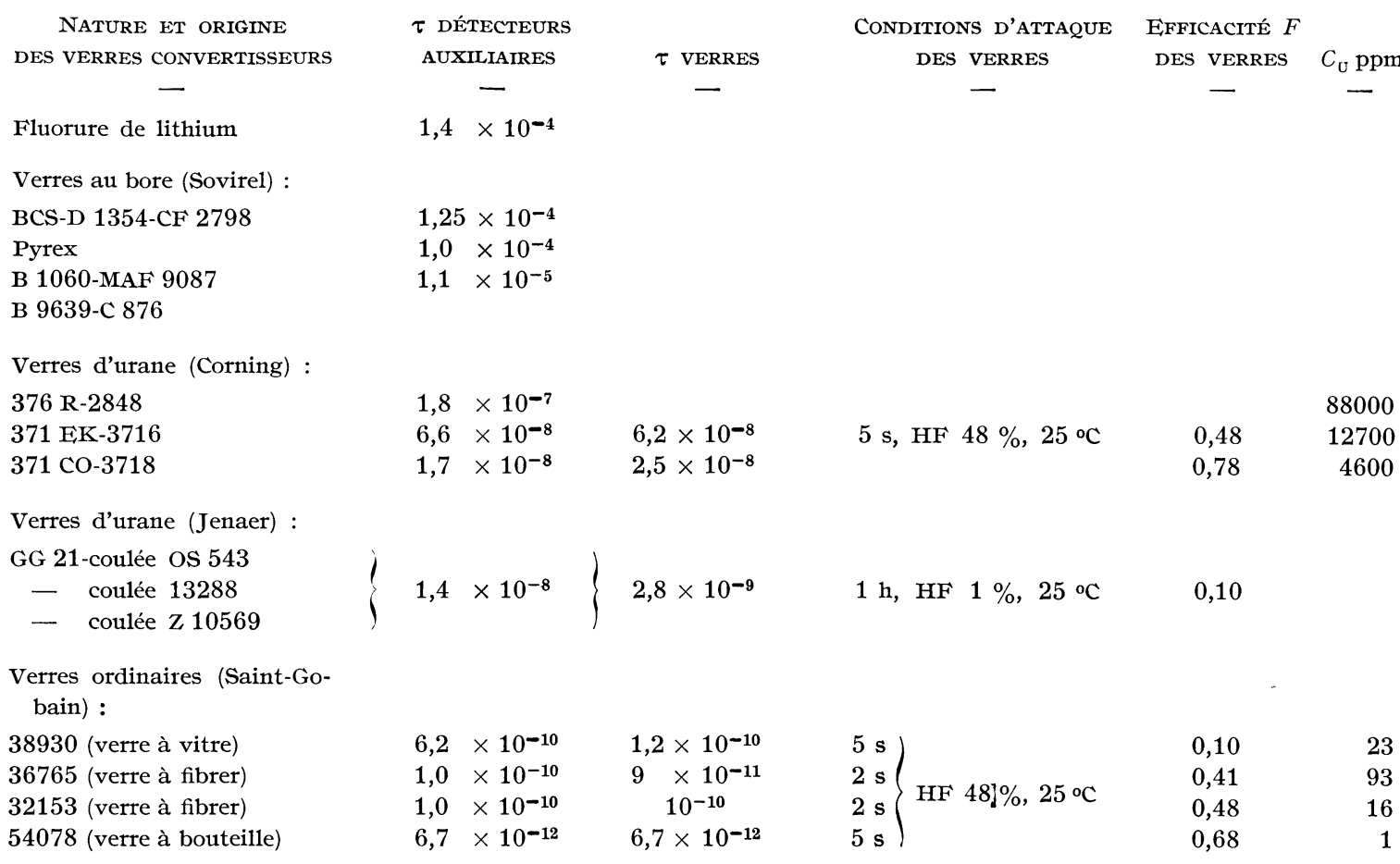

Verres à faible teneur en uranium :

1) Sovirel (verres d'optique):

C 0538-coulée P 680 coulée inconnue C 2036 (1-5-63)

2) Quartz et silice :

Quartz fondu, numéro de coulée non communiqué

Récapitulation des principaux résultats obtenus :

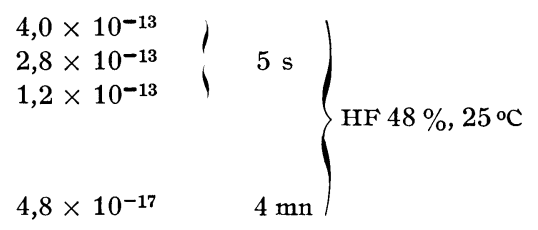

- Les détecteurs auxiliaires sont :

a) Pour le fluorure de lithium et les verres au bore : le nitrate de cellulose (attaque : $15 \mathrm{mn}$, dans $\mathrm{KOH} 20 \mathrm{~N}$ à $25^{\circ} \mathrm{C}$ ) ;

b) Pour les verres d'urane et les verres ordinaires : le mica muscovite (attaque : $15 \mathrm{mn}, \mathrm{HF} 48 \%, 25{ }^{\circ} \mathrm{C}$ ).

- Les valeurs portées dans la colonne 7 sont celles des concentrations communiquées par les fabricants.

et un noyau de recul ${ }^{7} \mathrm{Li}$ de $1 \mathrm{MeV}$. Dans les deux cas, les deux particules sont émises à $180^{\circ}$ l'une de l'autre, et on obtient ainsi une trace par réaction.

Le verre sert ainsi de convertisseur de neutrons, et, dans certains cas, de détecteur. Nous donnons dans le tableau I la liste des différents verres utilisés.

Pour mesurer les fluences supérieures à $10^{10} \mathrm{n} / \mathrm{cm}^{2}$, nous avons exposé aux neutrons thermiques de petits morceaux de verre $\left(\simeq 100 \mathrm{~mm}^{3}\right)$ contenant de l'uranium, dont l'une des faces était recouverte d'une lamelle de mica dont la concentration en uranium était beaucoup plus faible que celle du verre. Après irradiation, les traces des fragments de fission produits par les interactions des neutrons avec les atomes d'uranium 235 répartis dans le volume du verre sont révélées par une attaque fluorhydrique, d'une part sur des surfaces internes du verre, obtenues par fracture, d'autre part sur la surface du mica.

Pour déterminer les fluences inférieures à $10^{10} \mathrm{n} / \mathrm{cm}^{2}$, nous avons utilisé, comme convertisseur, des plaques de verre contenant de l'oxyde de bore (du verre Pyrex en particulier) et, comme détecteur, des feuilles de nitrate de cellulose de $0,1 \mathrm{~mm}$ d'épaisseur, fabriquées par la Poudrerie Nationale de Bergerac. Les traces, dues aux particules $\alpha$ et aux noyaux de recul enregistrés dans le nitrate, sont révélées au moyen d'une attaque potassique (solution $20 \mathrm{~N}$ ) de $15 \mathrm{mn}$ à $25^{\circ} \mathrm{C}$. 
Galibration des Dosimètres. - Nous avons tout d'abord réuni une centaine de types de verres différents, afin de sélectionner ceux dans lesquels les concentrations en uranium et en bore étaient très différentes d'un type à l'autre, mais très homogènes dans les échantillons d'un même type. Les irradiations aux neutrons thermiques ont eu lieu dans le réacteur ZOE (C.E.N., Fontenay-aux-Roses) pour les fluences inférieures à $10^{16} \mathrm{n} / \mathrm{cm}^{2}$, dans le réacteur EL3 (C.E.N., Saclay) pour les fluences supérieures à $10^{16} \mathrm{n} / \mathrm{cm}^{2}$.

Nous avons exposé tous ces verres, à l'exception des 8 verres contenant de l'oxyde d'uranium, dans une fluence de neutrons thermiques mesurée soigneusement par trois méthodes différentes : pour la première, nous avons obtenu une valeur $\varphi=(5,5 \pm 0,7) \times 10^{16} \mathrm{n} / \mathrm{cm}^{2}$, en mesurant la densité des traces de fission dans une lamelle de verre, calibrée à $\pm 15 \%$ par Fleisher et al. [7]; la deuxième détermination, $\varphi=(4,5 \pm 0,3) \times 10^{16} \mathrm{n} / \mathrm{cm}^{2}$, a été déduite de l'activité d'un fil en alliage AlCo; la dernière valeur, $\varphi=(4,7 \pm 0,5) \times 10^{16} \mathrm{n} / \mathrm{cm}^{2}$, nous a été donnée par R. Rentien, qui avait relevé la carte du flux, dans le canal du réacteur EL3 utilisé, en mesurant l'activité induite dans des feuilles d'or qui y avaient été exposées.

Après irradiation, nous avons attaqué tous les verres et sélectionné ceux où les densités de traces homogènes et mesurables avec précision et rapidité (c'est-à-dire comprises entre $10^{4}$ et $\left.6 \times 10^{6} \mathrm{t} / \mathrm{cm}^{2}\right)$; du fait de la mesure de $\varphi$, ces verres étaient calibrés. En effet, la densité de traces de fission induites, $\rho$, mesurée sur une face interne, est donnée par la relation suivante :

$$
\rho=N C R F \sigma \varphi=\tau \varphi
$$

où $N$ : est le nombre total d'atomes par $\mathrm{cm}^{3}$ de verre,

$C:$ la concentration atomique en uranium 235,

$R$ : l'épaisseur de la couche " effective », de l'ordre de $10 \mu$, à l'intérieur de laquelle chaque fission donne une trace révélable qui traversera le plan d'observation exposé ultérieurement à l'action de l'acide; dans le cas où le comptage est effectué sur une face externe, par exemple celle du détecteur de mica (dont le seuil de sensibilité d'enregistrement est voisin de celui du verre), $R$ est à diviser par 2 ,

$F$ : la proportion des fragments de fission traversant le plan d'observation qui sont effectivement comptés comme traces,

$\sigma=580$ barns : la section efficace de fission induite par les neutrons thermiques dans ${ }^{235} \mathrm{U}$.

Connaissant $\rho$ et $\varphi$, il nous était alors possible de déduire les valeurs de $\tau$ pour les verres sélectionnés. Après cette calibration, les verres contenant le plus de traces, donc la plus forte concentration en uranium, ont été utilisés, lors d'une irradiation dans une fluence approximative de $10^{13} \mathrm{n} / \mathrm{cm}^{2}$, pour étalonner ceux des verres d'urane qui contenaient le moins d'uranium. Ces derniers, à leur tour, lors d'une exposition dans un flux encore plus faible, ont permis la calibration des verres d'urane contenant le plus d'uranium, etc. En utilisant cette technique de calibration " de proche en proche ", nous avons pu déterminer les valeurs de $\tau$ pour chacun de nos dosimètres, en particulier ceux à traces $\alpha$ et les quartz fondus. Pour les quartz fondus, dans lesquels les concentrations en uranium sont très faibles, il nous a fallu choisir, après la première irradiation en flux connu, les verres qui contenaient le moins de traces, etc.

Dans le tableau I, nous avons rassemblé les caractéristiques de nos dosimètres : dans la colonne 1, nous avons porté le type, la provenance et le numéro de coulée de chacun des verres que nous avons sélectionnés; dans les colonnes 2 et 3 , la moyenne arithmétique des trois valeurs de $\tau$, d'une part pour le verre, d'autre part pour le détecteur auxiliaire; dans la colonne 4, les conditions d'attaque pour la révélation des traces; dans la colonne 5, la valeur de l'efficacité d'enregistrement $F$ des fragments de fission dans le verre, par rapport à celle mesurée dans le mica $\left({ }^{3}\right)$; dans la colonne 6 , les valeurs de la concentration en uranium dans les verres, calculées au moyen de la relation (1).

II. Remarques concernant l'utilisation des dosimètres. - 1. IDENTIFIGATION ET GOMPTAGE DES TRACES. - Dans le mica et le nitrate de cellulose, les traces révélées apparaissent sous forme de canaux creux approximativement cylindriques dont l'axe est confondu avec la direction de la trajectoire primaire. Quand leur longueur est supérieure à $2 \mu$, il est très facile de les différencier des autres défauts, car elles présentent un aspect très caractéristique de " comètes " (fig. 1). Nous avons obtenu des mesures de densité très reproductibles d'un observateur à l'autre en ne comptant que les comètes de longueurs supérieures à $2 \mu$, qui apparaissent après une durée de révélation normale.

Dans les verres, les traces sont révélées sous forme de figures de corrosion d'aspect moins caractéristique. Cependant, tant que la durée de l'attaque n'est pas trop prolongée, ces figures sont facilement discernables des autres défauts : elles sont en général elliptiques et pénètrent assez profondément dans le verre en suivant étroitement l'axe de la trajectoire primaire (fig. $2 \mathrm{~b}$ ). (Ceci n'est plus vrai lorsque les verres contiennent beaucoup de petites bulles; il est alors nécessaire de ne dénombrer comme trace que les figures de corrosion très elliptiques, car dans les verres artificiels les bulles sont en général sphériques.

(3) Pour obtenir cette valeur, il suffit de faire le rapport des densités de traces mesurées sur une face interne du verre et sur celle du détecteur auxiliaire de mica, pour lequel nous avons déterminé la valeur de $F$ par ailleurs [4]. 


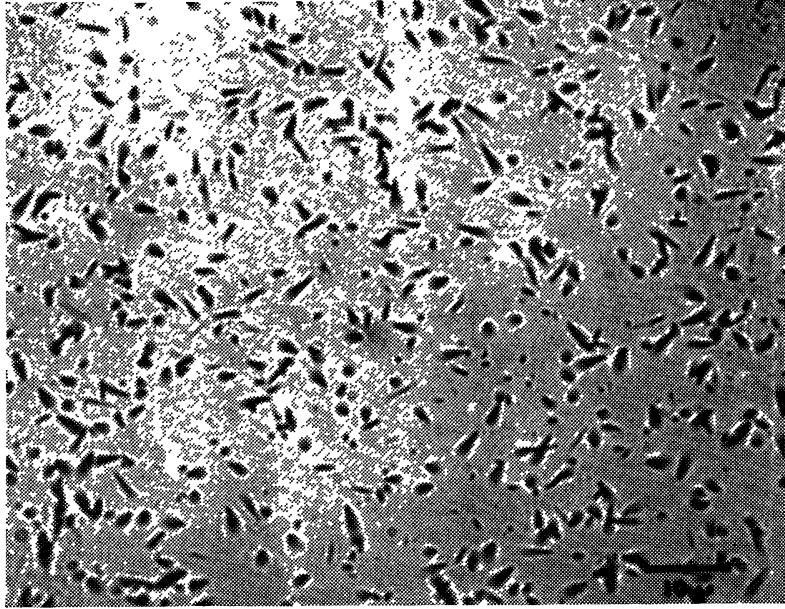

a

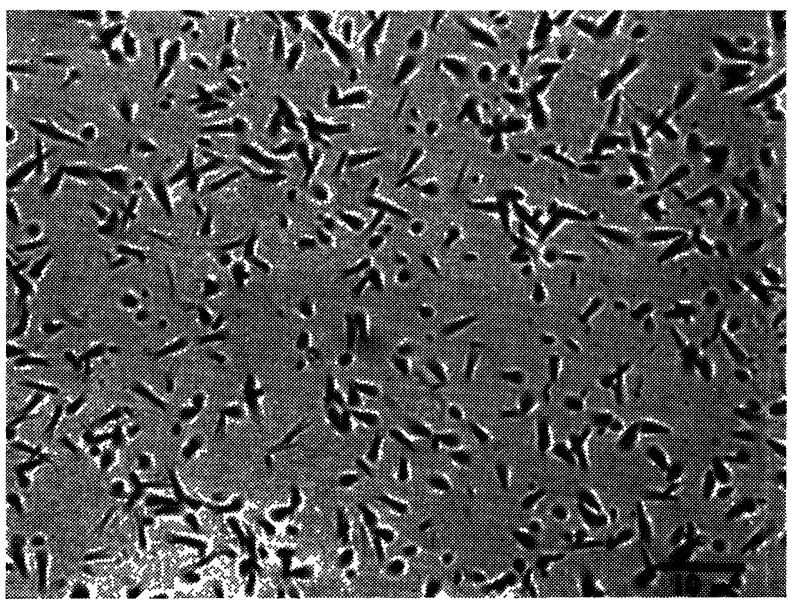

$\mathrm{b}$

FIG. 1. - Traces $(\alpha+$ noyau de recul) enregistrées dans le nitrate de cellulose :

1 a) Convertisseur : verre au bore D 1354 .

$1 \mathrm{~b})$ Convertisseur : fluorure de lithium.

Dans les verres que nous avons sélectionnés, la densité de telles bulles est très faible $\left(<100 / \mathrm{cm}^{2}\right)$, et par conséquent cette précaution n'est pas nécessaire.)

Mais il est parfois nécessaire de modifier les conditions d'attaque pour obtenir de telles figures. Ainsi, dans le cas du verre GG 21, en utilisant de l'acide fluorhydrique à $50 \%$, nous avons obtenu des figures de corrosion de qualité médiocre, ayant une très faible profondeur, pour lesquelles $F \simeq 0,08$ ( fig. $2 \mathrm{a}$ ); nous avons alors constaté qu'il suffisait d'utiliser un acide fluorhydrique moins concentré $(\simeq 1 \%)$ pour obtenir des figures de corrosion d'aspect normal (fig. $2 \mathrm{~b}$ ) pour lesquelles $F \simeq 0,35$. Nous avons constaté par ailleurs [11] que tous les paramètres d'attaque (nature du solvant, concentration, agitation ultrasonique, etc.) étaient susceptibles de modifier la vitesse et même la nature des processus physicochimiques conduisant à la révélation d'une trace. Dans les verres, nous avons fait des comptages sur des surfaces internes obtenues par fracture, et ce pour trois raisons :

a) On élimine tout risque de contamination des surfaces détectrices; ceci est important quand on veut mesurer des valeurs de fluences $\varphi>10^{16} \mathrm{n} / \mathrm{cm}^{2}$;

b) Nous avons constaté que, sur une surface externe, la densité de traces augmentait d'une manière complexe en fonction de la durée de l'attaque; en particulier, la valeur initiale ne correspond pas à une épaisseur effective égale à $R / 2$;

c) On peut obtenir des surfaces internes de très bonne qualité optique, en suivant la méthode que nous a indiquée $G$. Wagner : on obtient par sciage ou meulage un prisme à section triangulaire dont l'un des angles est voisin de $70^{\circ}$; en donnant un léger coup de marteau sur l'arête correspondant à cet angle, on obtient des lamelles très fines, à faces sensiblement parallèles.

2. Garactéristiques des répartitions en uRanium ET EN BORE DANS LES VERRES. - Les concentrations en uranium varient peu dans le groupe des 80 verres « ordinaires » que nous avons étudiés; en effet, nous n'en avons trouvé que 5 où les concentrations en uranium étaient nettement supérieures ou nettement inférieures à quelques parties par million. Dans les quartz fondus, les concentrations en uranium étaient très faibles et nous avons obtenu des densités de traces $\left(\simeq 100 / \mathrm{cm}^{2}\right)$ difficilement mesurables avec précision; par conséquent, l'incertitude sur les valeurs correspondantes de $\tau$ est plus élevée que pour les autres verres. L'association des verres 38930 et GG 21 est importante, puisqu'elle permet de relier le groupe des verres ordinaires au groupe des verres d'urane; de même, la liaison entre les dosimètres à traces $\alpha$ et les verres d'urane a pu être établie au moyen des verres $376 \mathrm{R}$ et 139639 ; la calibration du quartz fondu a été rendue possible grâce au verre CO 538 . Dans un verre donné, les concentrations en uranium ou en bore sont très homogènes. Cependant, dans le cas des quartz fondus, du fait des faibles densités de traces que nous avons observées, nous ne pouvons être certains d'une telle homogénéité; sur la figure 2 c, on peut voir une distribution de traces très inhomogène dans un type de quartz que nous avons dû renoncer à utiliser pour cette raison.

Il nous semble important de faire remarquer que, pour un même type de verre, les concentrations en uranium peuvent varier fortement d'une coulée à une autre. Par conséquent, si l'on veut qu'un dosimètre puisse être reproduit par d'autres expérimentateurs, il est indispensable de préciser, pour chaque verre, le numéro de la coulée. 
3. Limites D'emploi du Nitrate de Gellulose. Nous avons constaté que les interactions des neutrons avec les éléments constitutifs du nitrate de cellulose conduisent à la formation de traces révélables. Le taux de production de ces traces est assez élevé $\left(\tau=1,73 \times 10^{-6}\right)$; il est cependant suffisamment faible pour ne pas perturber les mesures de fluences, tant que $\varphi<10^{10} \mathrm{n} / \mathrm{cm}^{2}$.

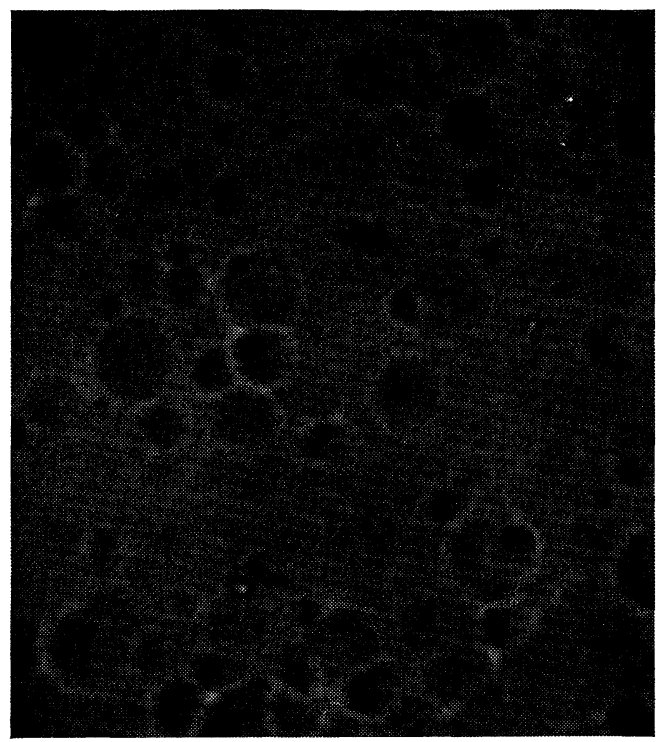

a

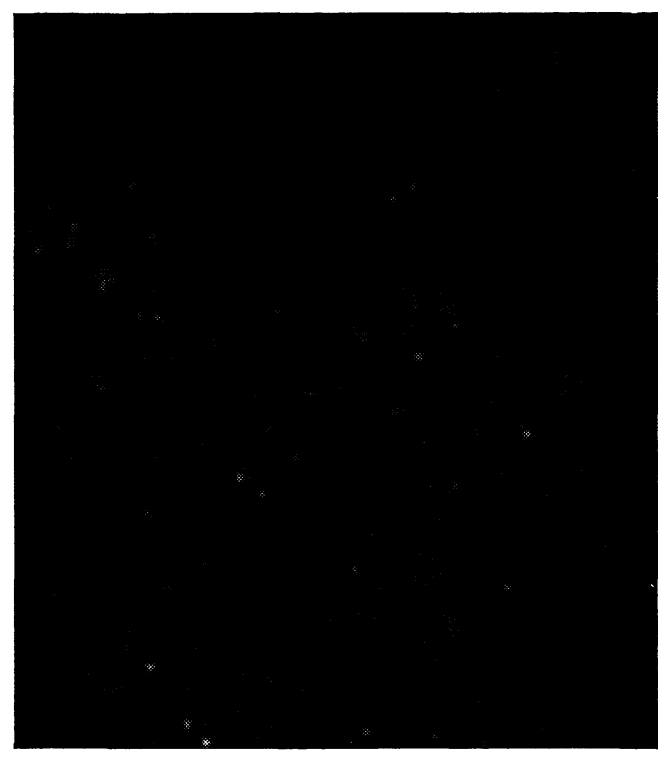

b
Une limitation plus sérieuse provient du fait que l'efficacité d'enregistrement des particules $\alpha$ dans le nitrate varie d'une fabrication à une autre. En particulier, dans deux feuilles de nitrate, de mêmes caractéristiques apparentes, découpées dans deux rouleaux fabriqués par la même compagnie, mais à 6 mois d'intervalle, l'efficacité d'enregistrement variait d'un facteur 2. Par conséquent, nous pensons qu'il faut calibrer chaque rouleau de nitrate, avant utilisation.

4. RadioAGTIVITÉ INDUITE DANS LES ÉGHANTILlONS. - Nous avons mesuré le débit d'équivalent de dose au contact (en $\mathrm{mRem} / \mathrm{h}$ ) d'échantillons de verre de $300 \mathrm{mg}$, en fonction de la fluence de neutrons thermiques $\varphi$ et de la durée $t$ écoulée depuis leur sortie de pile. Les résultats de nos mesures sont portés dans le tableau II, pour deux valeurs de fluences et deux valeurs de $t$.

Si l'on peut admettre, pour une manipulation brève, un débit d'équivalent de dose de $200 \mathrm{mRem} / \mathrm{h} \mathrm{(4)}$, on voit que, une semaine après leur sortie de pile, tous les verres peuvent être manipulés sans précautions excessives dans leur domaine d'application $\left(^{5}\right)$, à l'exception de ceux destinés à la mesure des fluences $\varphi \geqslant 10^{18} \mathrm{n} / \mathrm{cm}^{2}$. Avant d'utiliser ces derniers, il est préférable d'attendre deux semaines, et d'opérer sur de petits échantillons $(\simeq 10 \mathrm{mg}$ ) que l'on obtient par

(4) R. Pannetier, vade mecum du Technicien, 1968, p. E9.

${ }^{(5)}$ La valeur de $\varphi_{1}$ est 1000 fois plus élevée que celle du flux maximum qui peut être mesuré avec les verres d'urane GG 21.

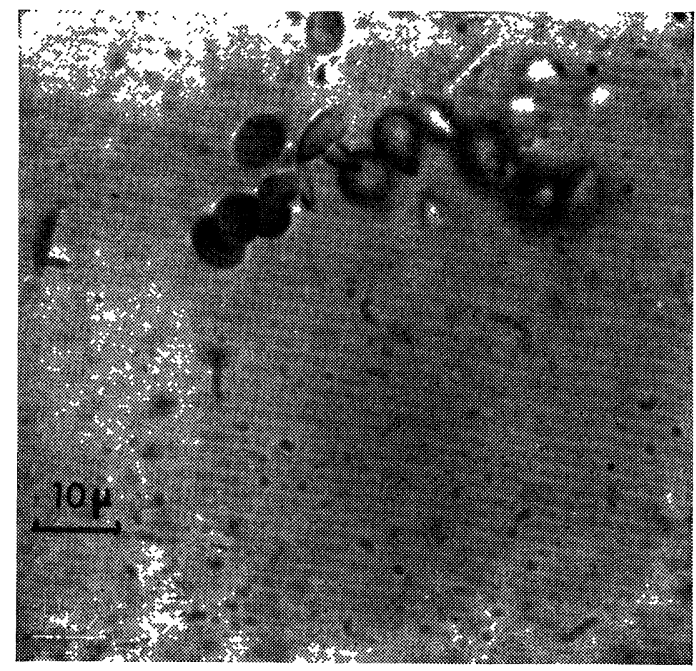

FIG. 2. - Influence de la concentration en acide fluorhydrique sur l'aspect des traces dans le verre GG 21 :

2 a) Attaque par $\mathrm{HF} 48 \%$.

2 b) Attaque par $\mathrm{HF} 1 \%$.

2 c) Traces révélées dans un échantillon de quartz fondu. 
TABLEAU II

\begin{tabular}{ccc} 
& \multicolumn{2}{c}{$\varphi_{1}$} \\
\cline { 2 - 3 } TyPE DE VERRE & $A\left(t_{1}\right)$ & $A\left(t_{2}\right)$ \\
- & - & - \\
Verre d'urane & & \\
371 CO & 1,8 & 0,3 \\
GG 21 & 2,7 & 0,3
\end{tabular}

Verre à bouteille 54078

Verres d'optique à faible teneur en uranium

C 0538

C 2036

825

420

Quartz fondu

Débits d'équivalents de dose au contact de différents verres, en fonction de la fluence de neutrons thermiques et du temps $t$ écoulé depuis leur sortie de pile :

$$
\begin{aligned}
& \varphi_{1}=4,1 \times 10^{15} \mathrm{n} / \mathrm{cm}^{-2} \\
& \varphi_{2}=5 \times 10^{17} \mathrm{n} / \mathrm{cm}^{-2} \\
& t_{1}=1 \text { semaine } \\
& t_{2}=2 \text { semaines. }
\end{aligned}
$$

fracture dans une pochette plastique. On peut alors étendre les mesures de fluences jusqu'à $10^{21} \mathrm{n} / \mathrm{cm}^{2}$, puisque le débit d'équivalent de dose de $10 \mathrm{mg}$ de quartz fondu serait inférieur à $5 \mathrm{mRem} / \mathrm{h}$. Il n'en reste pas moins que ces échantillons, en dehors de leur exploitation, devront être stockés dans un lieu protégé, pour éviter tout risque d'irradiation permanente.

Nous avons également constaté que l'activité induite ne dépend pas de la concentration en uranium, puisque le quartz fondu contient $10^{5}$ fois moins d'uranium que le verre 54078; elle n'est donc pas due à la décroissance des produits de fission. Cette conclusion est encore renforcée par le fait que les courbes de décroissance sont en général différentes d'un type de verre à l'autre, ce qui implique l'existence d'éléments activés différents.

III. Caractéristiques des dosimètres. - 1 . SensiBILITÉ. - Sur la figure 3, nous avons représenté les gammes de fluences que l'on peut mesurer avec quelques-uns des dosimètres dont les caractéristiques sont portées dans le tableau I. On voit que l'on peut mesurer facilement des valeurs de $\varphi$ comprises entre $10^{6}$ et $10^{23} \mathrm{n} / \mathrm{cm}^{2}$. Il est possible, en général, de choisir un verre où la densité de traces sera comprise entre $10^{5}$ et $6 \times 10^{6} / \mathrm{cm}^{2}$; il faut alors moins de $10 \mathrm{mn}$ pour dénombrer 1000 traces et ainsi avoir une faible erreur statistique de comptage.

Cependant, nous pensons qu'il est préférable de limiter la valeur supérieure de $\varphi$ à $10^{21} \mathrm{n} / \mathrm{cm}^{2}$, car l'activité induite dans le dosimètre deviendrait dan-

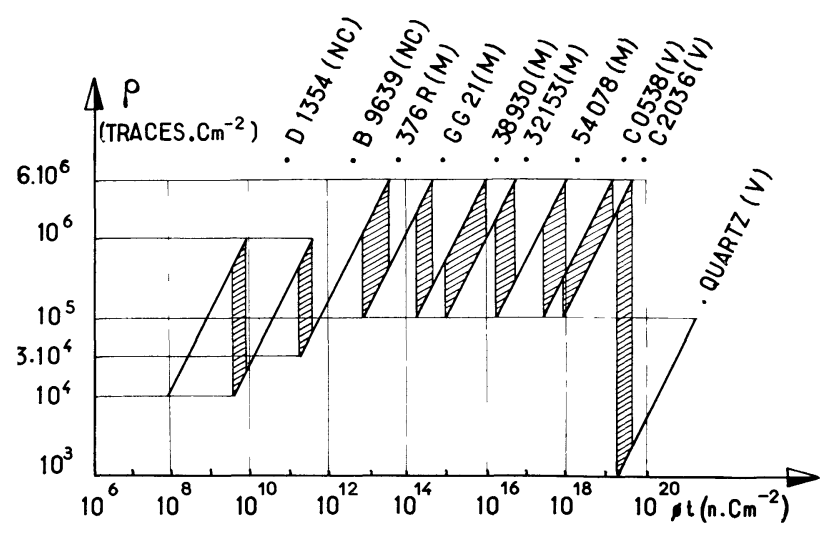

Fig. 3. - Domaines d'application de quelques-uns des verres proposés :

$(\mathrm{NC}):$ détecteur $=$ nitrate de cellulose,

(M) : détecteur = mica muscovite,

(V) : détecteur $=$ verre convertisseur lui-même.

En grisé : bandes de recouvrement entre les verres successifs.

gereuse. De plus, quand $\varphi>10^{20} \mathrm{n} / \mathrm{cm}^{2}$, on ne peut plus négliger l'appauvrissement en ${ }^{235} \mathrm{U}$ ni la formation de ${ }^{239} \mathrm{Pu}$ à partir de ${ }^{238} \mathrm{U}$; on peut alors montrer qu'en utilisant les valeurs de $\tau$ non corrigées portées dans le tableau I, on fait une erreur de $2 \%$ quand $\varphi=10^{20} \mathrm{n} / \mathrm{cm}^{2}$ et de $6 \%$ quand $\varphi=10^{21} \mathrm{n} / \mathrm{cm}^{2}$.

2. PRÉcision des dÉterminations. - La précision des mesures absolues de flux dépend essentiellement de celle concernant les valeurs de $\tau$. En effet, l'erreur statistique sur $\rho$ peut être rendue très faible en choisissant le verre approprié à la gamme de fluences que l'on veut utiliser et, comme nous l'avons déjà dit, nous avons sélectionné des verres où les répartitions en éléments convertisseurs étaient très homogènes. Comme les valeurs de $\tau$ ont été déterminées par la mesure de la fluence à laquelle ont été exposés les verres ordinaires - contenant quelques parties par million d'uranium -, nous voyons que l'incertitude sur les valeurs absolues de flux, que nous évaluons à $\pm 10 \%$, est essentiellement celle des autres méthodes utilisées pour mesurer le flux d'étalonnage.

Par suite de la méthode de calibration adoptée (chaque verre a été étalonné par rapport à un autre verre), la précision sur les mesures relatives de fluences est excellente et doit être de l'ordre de quelques pour cent. Ceci pourrait être intéressant pour les spécialistes de l'état solide qui étudient les effets physiques des neutrons en fonction de la dose d'irradiation ou pour les géochronologistes, qui essayent, au moyen de la méthode développée par Price et Walker [12], de mesurer des variations éventuelles d'âges géologiques dans des minéraux contenant des concentrations en uranium très différentes.

3. Avantages des Dosimètres. - Les dosimètres que nous avons construits présentent les avantages 
suivants, par rapport à ceux de même type construits précédemment :

1) Nous avons choisi un même matériau convertisseur, le verre, dans lequel les concentrations en uranium et en bore sont très homogènes; la gamme de fluences couverte avec ce convertisseur est très large et les mesures sont très reproductibles;

2) Le prix du verre est beaucoup plus faible que celui de feuilles d'uranium, enrichies ou non en ${ }^{235} \mathrm{U}$;

3) La résistance mécanique des surfaces de verre est très supérieure à celle de couches minces évaporées d'uranium ou d'oxyde de bore; alors qu'on ne peut en général utiliser ces couches qu'une fois, nous avons effectué plus de 20 déterminations de fluences, en utilisant des détecteurs auxiliaires placés sur les mêmes plaquettes de verre GG 21 .

4) La précision des mesures relatives de fluences est excellente.
Les avantages de la dosimétrie par traces de fission ont déjà été soulignés par ailleurs [3-10]; rappelons simplement que la température et les rayonnements faiblement ionisants produits dans les réacteurs nucléaires ont une influence négligeable sur leur réponse.

Remerciements. - Nous devons beaucoup au Docteur P. Le Glerc, de la direction des Recherches de la Compagnie Saint-Gobain, qui nous a procuré la plupart de nos échantillons de verre, soit en nous les envoyant directement, soit en nous adressant aux Services de Recherches d'autres Compagnies. Nous remercions Mme Vagner, de la Compagnie Sovirel, et M. J. F. Wosinski, Corning Glass Works, New York, pour nous avoir adressé des échantillons de verres d'urane et de verres de bore avec la plupart de leurs caractéristiques techniques. Nous avons également bénéficié de l'intérêt actif et des critiques des Professeurs Y. Gauchois et R. M. Walker.

\section{BIBLIOGRAPHIE}

[1] SILK (E. C. H.) et BARNes (R. S.), Phil. Mag., 1959, 4, 970 .

[2] Price (P. B.) et Walker (R. M.), J. Appl. Phys., 1962, 33, 3407.

[3] Walker (R. M.), Price (P. B.) et Fleisher (R. L.), Appl. Phys. Letters, 1963, 3, 28.

[4] Debeauvais (M.), Maureitute (M.), Mory (J.) et WALKER (R. M.), Inter. J. Appl. Radiations and Isotopes, 1964, 15, 289.

[5] Mory (J.), Rapport C.E.A., 1965, no 2846.
[6] Prevo (P.), Dahi (R. E.) et Yoshikawa (H. H.), J. Appl. Phys., 1964, 35, 2636.

[7] Fleisher (R. L.), Price (P. B.) et WALker (R. M.), Nucl. Science and Eng., 1965, 22, 153.

[8] BECKER (K.), USNRDL-TR-904, 1965.

[9] Tuys (J. W. N.), Nuclear Application, 1967, 3, 372.

[10] Fleisher (R. L.), Price (P. B.) et WALKer (R. M.), Rev. Scient. Inst., 1966, 37, 525.

[11] Maureit'te (M.), Thèse Doctorat d'État, Paris, 1965.

[12] Price (P. B.) et WALker (R. M.), J. Geoph. Res., 1963, 68, 4847. 